One of the principles on which the Health Service is founded, and which the medical profession has accepted since 1945, ${ }^{6}$ is that it should be available to the whole population. In other words it is not merely for the indigent, let alone the destitute. A second principle is that within the National Health Service the nature and quality of the services offered to patients are in accordance with his medical needs irrespective of his financial status. A corollary of this provision is that the Health Service should not be financed in such a way as to impose a financial deterrent between the patient and the treatment his condition requires. And a third principle is that nobody is forced to use the N.H.S. The continuance of private practice means that there is something other than a State service for patients who prefer it.

What seemed in the Beveridge era to be the relatively simple task of providing a service on which the demands would steadily diminish from an ever healthier population has proved to be a political hot potato. The population undoubtedly is healthier in measurable ways than it was a quarter of a century ago. And in this improvement the Health Service has played its part, as have the general increase in the standard of living and the discovery and marketing of new drugs mainly by the pharmaceutical industry. But medical discoveries and the cost of translating them into clinical practice seem to move faster than the country's general economy-a disparity not confined to Great Britain. If the Health Service is to keep pace with the nation's needs a review of its financing in the New Year should be encouraged. But two points are worth emphasizing. Firstly, only the nation can create the wealth from which the Health Service is to be financed. The welfare services are not a rock against which the people can lean, but an air-cushion that will grow or diminish in accordance with the exertions of the nation itself. Secondly, the guiding principles of good health care must be kept to the forefront, and above all the provision of patients with medical care in accordance with their needs.

\footnotetext{
1 B.M.A. Advisory Panel, Health Services Financing. London, British Medical Association, 1970.

2 Aitken, Sir R., British Medical fournal, 1970, 2, 229.

3 Lees, D., British Medical fournal, 1970, 4, 292.

The Times, 21 December 1970

Barnes, S., Sunday Times, Colour Supplement, 29 November 1970.

- British Medical fournal, 1945, 1, 668 .
}

\section{Enteroviral Disease}

The existence of a group of enteric viruses has emerged largely as a result of research into poliomyelitis and related infections. Before 1949 the study of poliomyelitis was restricted to experimental studies in primates. Even with such laborious and expensive procedures three immunological types of poliovirus had by then been identified. But a breakthrough lay just ahead.

In 1948 G. Dalldorf and G. M. Sickles ${ }^{1}$ isolated a new virus from the faeces of two boys suffering from paralytic poliomyelitis. The following year additional types of virus and the first of what later became known as the group B viruses were isolated. Subsequent studies disclosed a group of these agents, termed Coxsackie viruses from the town from which the first isolations were made. They could be broadly classified into two groups, A and B, by the histopathological lesions they caused in infected immature animals, either mice or hamsters. Both group A and group B Coxsackie viruses were found to consist of many serological subtypes-24 group A strains and 6 group B. Clinical epidemiological studies showed a close association of group A strains with herpangina and later with a similar condition characterized by nodular lesions on the uvula and pharynx described by A. J. Steigman and colleagues ${ }^{2}$ as acute lymphonodular pharyngitis.

Coxsackie A strains have also been identified in cases of hand-foot-mouth disease, undifferentiated febrile illness, and some cases of coryza. Coxsackie A7 and A9 strains have been found in cases of paralytic disease, occasionally with poliovirus present as well, and also in cases of aseptic meningitis but not to the same extent as the group B strains.

Coxsackie B strains have been identified as one of the most important causes of aseptic meningitis and also as causing Bornholm disease, myocarditis, pericarditis, and a fulminating systemic infection of the newborn characterized by myocarditis, meningoencephalitis, and often lesions of the liver. ${ }^{3}$

In $1949 \mathrm{~J}$. F. Enders and his colleagues ${ }^{4}$ showed that poliovirus could be grown in cultures of non-neural tissues. Their work had far-reaching implications and was to affect virtually every aspect of virology. As a direct outcome of it tissue and cell cultures were reintroduced, first for research on animal viruses and then for the standard laboratory diagnosis of virus disease. In this way a third group of viruses was identified. Some were recovered from the human intestinal tract which were neither pathogenic for monkeys nor for immature animals and could be isolated only in cell cultures. These agents were provisionally termed echoviruses (enteric cytopathogenic human orphan viruses), as they had at that time no clear association with human disease.

In the past 20 years much has been learned about the role in human disease of echoviruses, which consist of 30 antigenic types. In general they have been identified as causing infection in clinical syndromes in which other viruses, and bacteria too, may be found rather than as causing distinct diseases on their own. Echoviruses have been identified in sporadic cases and in outbreaks of aseptic meningitis. Outbreaks and sporadic cases of enteroviral disease with maculopapular rashes, with or without aspetic meningitis, have frequently been reported as due to these viruses, as have outbreaks of undifferentiated febrile disease.

Studies in recent years have shown that there is no clear distinction between many clinical syndromes usually attributed to the three principal enteric viruses-poliovirus, Coxsackie A and $B$, and the echoviruses. Poliovirus remains the principal cause of paralytic disease, but both Coxsackie and echoviruses can also rarely cause paralytic disease and encephalitis. Rashes, lymphadenopathy, myalgia, Bornholm-like disease, pericarditis, and myocarditis can also be associated with echovirus. Strains of echovirus $(11,14$, and 18) have been isolated in several outbreaks of diarrhoea in children, but though the isolation rate has been somewhat higher in cases of diarrhoea than in control groups a clear-cut aetiological relationship has yet to be established. Furthermore, the very severe form of acute gastroenteritis seen in infants, associated with severe vomiting and diarrhoea and requiring fluid replacement, is not a feature of enteroviral disease.

Reports of two recent outbreaks are of special interest. In 1967-8 an extensive epidemic of herpangina occurred in infants and young children in the Federal Republic of Germany. ${ }^{5}$ Herpangina was the dominant symptom, but the nodular non-eroding form as well as the vesicular form was seen, as usual on the soft palate and palatine arch. Other prominent symptoms were follicular tonsillitis, fever, lymphadenopathy, interstitial lesions in the lungs, and bronchitis. 
Many of the patients had rashes resembling measles and Koplik spots; some had rubella-like rashes; cases of parotitis and orchitis were also seen. In the older children vomiting, meningitis, and encephalitis were notable. The incubation period was 2-11 days, the course of the disease about five days, but quite a number of patients had two or more recurrences after an afebrile interval-unusual in this type of infection. The age incidence was also unusual in that there was a high incidence of infection in infancy: 151 of the 590 patients were under 12 months old and 40 were under 3 months. This suggests that there was little basic immunity to the echovirus strain 30 which was isolated from a number of cases. Six of the children died, all having had symptoms of infection in the central nervous system, which was established at necropsy in three cases.

Recently A. S. Mary and J. H. Swallow ${ }^{6}$ have described an epidemic of echo-6-virus infection in Essex in the summer and autumn of 1969 . Of the 14 patients seven were adults and seven children. Eleven had aseptic meningitis of acute onset, with fever, headache, signs of meningeal irritation, and vomiting. Echo-6 virus was isolated from eight patients, including one with a normal cerebrospinal fluid. In addition one patient had myalgia, with bilateral lower chest pain; one had pericarditis; one had pharyngitis; and a 5-year-old boy had signs of nephritis. All patients recovered without sequelae.

1 Dalldorf, G., and Sickles, G. M., Science, 1948, 108, 61.

- Dalldorf, G., in Viral and Rickettsial Infections of Man, ed. F. L. Horsfal and I. Tamm, 4th edn. London, Pitman Medical Publishing Co., 1965.

Steigman, A. J., Lipton, M. M., and Braspennickx, H., fournal of Pediatrics, 1962, 61, 331.

- Enders, J. F., Weller, T. H., and Robbins, F. C., Science, 1949, 109, 85.

- Keuth, U., Esser, I., Wilhelmi, J., and Wilhelmi, I., German Medical Monthly, 1970, 15, 254.

- Mary, A. S., and Swallow, J. H., Postgraduate Medical fournal, 1970, 46, 265.

\section{Three Changes}

The B.M.f. enters 1971 with three main changes. The most obvious is a new layout; the most regrettable, because of its. cause-penal increases in postal charges-is a switch to lighter-weight paper; and the third is a rearrangement and expansion of our teaching sections under the general heading, Medical Practice.

The new layout is to the design of Mr. Ray Fishwick, and continues what he began three years ago when the B.M.f. changed its cover and went glossy. It aims to give the journal a clean, up-to-date appearance, and to make it easier to read and to set in print. On the cover visual emphasis is given to the B.M.Y.'s most important features-the leading at ticles, the reports of original work, on which a medical journal's reputation internationally so much depends, and Medical Practice. Eric Gill's emblem has gone, but the familiar grey remains. Inside, the layout is designed to ease the reader's task: leader references appear unambiguously at the end of the leader to which they belong; the titling of papers has been tidied up and detailed but essential information about authors put at the foot of the page. In certain of the three-column pages, such as the book review pages and the obituaries, the reader's way is more clearly marked than it was. There are also two changes in the recording of units of measurement- $\varnothing$ the International System of Units (SI), based on the metre $\stackrel{.}{.}$ and kilogramme, is adopted and the full point after the symbol $\overrightarrow{\bar{F}}$ for any unit of measurement is dropped, except in the case $\overrightarrow{0}$ of the litre (1.), where there could be confusion.

When the Post Office announced its proposals last July for $\frac{\overline{\bar{N}}}{\vec{D}}$ increased charges publishers of learned journals-like other $\stackrel{\Phi}{\mathscr{Q}}$ Post Office customers-found themselves facing heavy extra $\approx$ costs. Despite vigorous protest, however, the charges are to be $\vec{\circ}$ introduced (with minor alleviations only), and the rates will begin to go up shortly. Many journals will be hit hard, and $\vec{\omega}$ those that come out frequently and have a large circulation $\frac{}{5}$ will be hit hardest of all. The B.M. $\mathcal{Y}$. is a case in point. Last year its postage cost about $£ 200,000$. If its weight remained iv unchanged, the new charges over a full year would add another $£ 128,000$ to this already heavy bill. The only practicable answer, short of a crippling reduction in size, is to use a lighter paper. The one we have chosen is of good quality and we hope it $\vec{N}$ will prove acceptable.

The new section, Medical Practice, combines features from the previous Current Practice and Middle Articles. It brings together the journal's main contribution, outside the leading $\vec{\bullet}$ articles, to the continuing education of doctors. The section will contain both new and familiar features whose aim is to help practitioners keep pace with clinical advance. It will deal with the practice as much as the science of medicine, whether in the hospital, the health centre, or the home. Features appearing this week for the first time are a Thera- $\frac{\mathbb{D}}{\mathbb{2}}$ peutic Conference and the first of a new group of Unheard $\overrightarrow{\vec{P}}$ Voices-this time those of medical teachers. The Thera- $\frac{9}{3}$ peutic Conference comes by arrangement with Professor A. G. Macgregor, to whom we are indebted, from ward rounds held at the Department of Therapeutics and Pharmacology at Aberdeen University. Its subject is the management of hypertension, and it is the first of a series of recorded 0 discussions from Aberdeen on drug treatment. As well as refresher course articles, other familiar contributions to this $\delta$ section will be the Clinicopathological Conferences from the Royal Postgraduate Medical School, Clinical Progress articles on growing points of medicine, and Any Questions? No one $\stackrel{N}{\sigma}$ can practice medicine really effectively without decent $\rightarrow$ organization, and this also will be a matter for description in Medical Practice.

We are encouraged that in each of the last three years the paid circulation of the B.M.F. has risen by over 1,000 subscriptions. Last year the increase was 1,500 . We trust that the changes we are introducing this year will be to the liking of our readers, the many overseas as well as all those at home. 